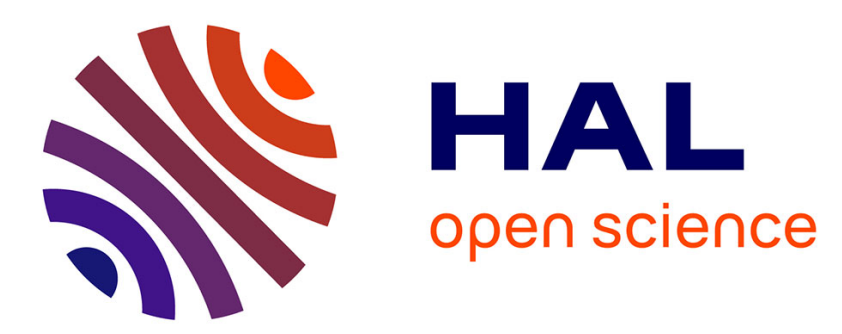

\title{
Passive control of a two degrees-of-freedom pendulum by a non-smooth absorber
}

\author{
Gabriel Hurel, Alireza Ture Savadkoohi, Claude-Henri Lamarque
}

\section{To cite this version:}

Gabriel Hurel, Alireza Ture Savadkoohi, Claude-Henri Lamarque. Passive control of a two degreesof-freedom pendulum by a non-smooth absorber. Nonlinear Dynamics, In press, 10.1007/s11071-01904891-0 . hal-02068520

\section{HAL Id: hal-02068520 \\ https://hal.science/hal-02068520}

Submitted on 5 Jun 2019

HAL is a multi-disciplinary open access archive for the deposit and dissemination of scientific research documents, whether they are published or not. The documents may come from teaching and research institutions in France or abroad, or from public or private research centers.
L'archive ouverte pluridisciplinaire HAL, est destinée au dépôt et à la diffusion de documents scientifiques de niveau recherche, publiés ou non, émanant des établissements d'enseignement et de recherche français ou étrangers, des laboratoires publics ou privés. 


\title{
Passive control of a two degrees-of-freedom pendulum by a non-smooth absorber
}

\author{
G. Hurel, A. Ture Savadkoohi, C.-H. Lamarque \\ Univ. Lyon, ENTPE, LTDS UMR 5513, rue Maurice Audin, 69518 Vaulx-en-Velin \\ Email: gabriel.hurel@entpe.fr
}

Accepted on March 13, 2019

\begin{abstract}
A pendulum, which can oscillate in two directions, is subjected to a generalized external force. A non-smooth absorber is coupled to the pendulum with an arbitrary location and orientation. The equations of the system are derived and are treated with a multiple scale method. At fast time scale, the topology of the slow invariant manifold is described with its stable and unstable zones. The equilibrium and singular points of the system are detected at the first slow time scale. The responses of the main system, given as a function of the frequency of the external force, show reductions of the vibration levels. The analytic predictions are compared by direct numerical time integration of the equations of the system. They illustrate the operationality of the non-smooth absorber in several cases.
\end{abstract}

Keywords Two degrees-of-freedom Pendulum, Non-smooth absorber, Multiscale method, fast/slow dynamics

\section{Introduction}

Many mechanical systems are subject to external or internal forces leading them to vibrate. For reasons of mechanical integrity, noise or comfort, absorbers are used to reduce the vibrations. Passive absorber have the advantage of not requiring any external input energy [1]. They can be linear or nonlinear. Whereas the linear absorbers are tuned on a single mode frequency $[2,3]$, the nonlinear absorbers can be efficient on a large frequency range. Roberson [4] added a cubic nonlinearity in the restoring force function of an originally linear absorber device, and so they widened the effective frequency range.

Since then, plenty of nonlinear absorbers have been designed. One of them called nonlinear energy sink (NES) [5, 6], has a purely nonlinear restoring force function (cubic nonlinearity in its original form) with no linear part. Its interactions with the main system lead to strongly modulated response (SMR) oscillations [7] or periodic regime [8]. It is possible to replace the polynomial nonlinearity by a non-smooth piecewise linear function [9-14]. Using the non-smooth nonlinearity for the absorber is interesting when the overall system is under pre-stressing terms, e.g. the system under a gravitational field [15]. Endowing polynomial nonlinearities for the absorber in such cases induces a linear restoring forcing terms due to the static equilibrium position of the system and destroys pure nonlinear nature of the absorber.

Here, the aim is to control the oscillations of a two degrees-of-freedom (dof) pendulum excited by an external force. Matsuhisa $[16,17]$ has proposed the control on a single dof pendulum by a linear absorber. Starovetsky [18] and Pham [19] have studied the control of a two dof system with a single NES in one dimension. Hurel et al. [20] analyzed a two dof pendulum coupled with a cubic restoring force NES. This article studies the control of 
a two dof pendulum by a single non-smooth NES located at a given position and oriented at an arbitrary direction. The considered academic system of this the paper can be applied to many realistic structures such as gondola lift.

The description of the main system and the non-smooth absorber is given in Sect. 2. Then, the dynamical equations of the system are written from Lagrange equations. In Sect. 3, the equations are studied analytically with a multiple scales method. The slow invariant manifold, the equilibrium and singular points are calculated. Numerical results are given in Sect. 4 in order to confirm and illustrate analytic results on three different cases. Finally, the paper is concluded in Sect. 5 .

\section{Studied system}

\subsection{The main system}

The studied system represented in Fig. 1 is a pendulum with the mass $M$, fixed at the point $O$, able to oscillate around the axes $\underline{e_{x}}$ and $e_{y}$ of the main reference frame $\left(\underline{e_{x}}, e_{y}, \underline{e_{z}}\right)$. A gravitational field $-g \underline{e_{z}}$ exists. The oscillations of the pendulum around the $\underline{e_{x}} \overline{\text { axis }}$ is subjected to a rotational constraint with rigidity as $K_{y}$. Its tensor of inertia $\mathbf{I}$ is diagonal in the local reference frame of the pendulum. The distance between the center of mass $G$ and the point $O$ is called $L$. Its coordinates in the main reference frame read:

$$
\left\{\begin{array}{l}
x_{G}=L \sin (\varphi) \\
y_{G}=L \sin (\psi) \cos (\varphi) \\
z_{G}=L \cos (\varphi) \cos (\psi)
\end{array}\right.
$$

A generalized force of amplitude $F_{0}$ and frequency $\Omega$ is applied on both dof of the pendu$\operatorname{lum}[\varphi, \psi]^{\mathrm{T}}$ distributed as follows:

$$
F_{(\varphi, \psi)}=F_{0} \sin (\Omega t)\left[\begin{array}{l}
\cos (\beta) \\
\sin (\beta)
\end{array}\right]
$$

\subsection{Coupled non-smooth absorber}

The pendulum is coupled to a non-smooth absorber of mass $m$ which is assumed to be much lower than $M$ :

$$
0<\frac{m}{M} \ll 1
$$

It is located on the line $O G$ at the distance $a$ from $O$ as seen on Fig. 3a. The restoring forcing function of the non-smooth absorber is given by a function $s(u)$ (see Fig. 2), where $u$ corresponds to the displacement of the mass $m$ :

$$
s(u)= \begin{cases}k(u+d) & \text { if } u<-d \\ 0 & \text { if }-d \leq u \leq d \\ k(u-d) & \text { if } u>d\end{cases}
$$

where $k$ and $2 d$ are respectively stiffness and clearance of the non-smooth oscillator. Practically, this absorber can be made with a mass $m$ guided between two linear springs of stiffness $k$ spaced from a distance $2 d$ (see Fig. 3b). The absorber is also characterized by a viscous damping of coefficient $C_{u}$.

In the reference frame linked to the pendulum $\left(\underline{e_{x}^{\prime}}, e_{y}^{\prime}, \underline{e_{z}^{\prime}}\right)$, the orientation of the nonsmooth absorber $\underline{e_{u}}$ is determined by a constant angle $\bar{\alpha}$ :

$$
\underline{e_{u}}=\cos (\alpha) \underline{e_{x}^{\prime}}+\sin (\alpha) \underline{e_{y}^{\prime}}
$$

In the main reference frame, the coordinates of the mass $m$ read: 


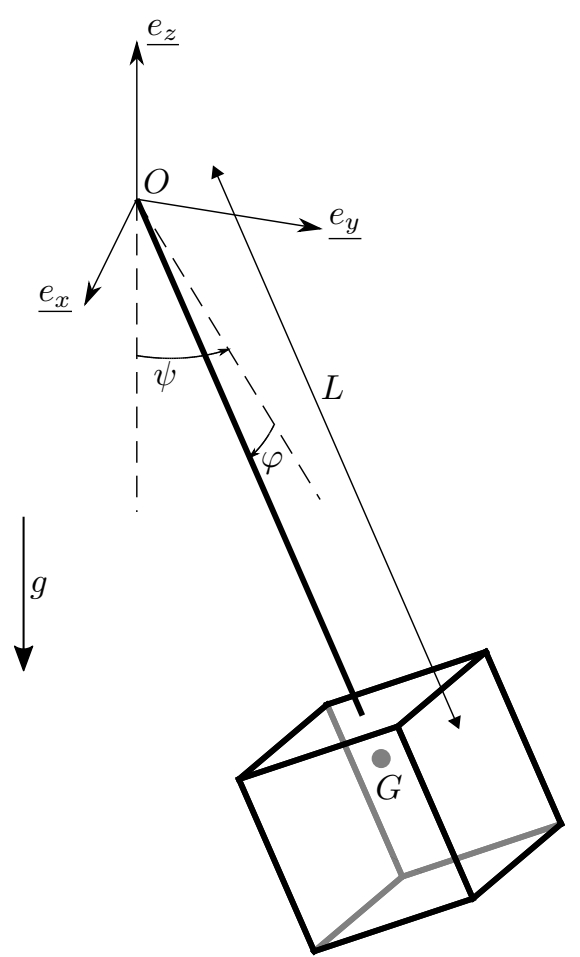

Figure 1: The pendulum clamped at the point $O$. It can oscillate around $\underline{e}_{x}$ and $e_{y}$ with generalized coordinates as $\varphi$ and $\psi$ respectively. $L$ is the distance between the center of mass $G$ and $O$.

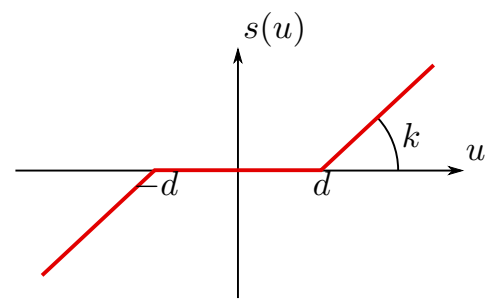

Figure 2: Diagram of the non-smooth restoring force $s(u)$ of the absorber

$$
\left\{\begin{array}{l}
x_{m}=a \sin (\varphi)+u \cos (\varphi) \cos (\alpha) \\
y_{m}=a \sin (\psi) \cos (\varphi)+u[\cos (\psi) \sin (\alpha)-\sin (\varphi) \sin (\psi) \cos (\alpha)] \\
z_{m}=-a \cos (\varphi) \cos (\psi)+u[\sin (\varphi) \cos (\psi) \cos (\alpha)+\sin (\psi) \sin (\alpha)]
\end{array}\right.
$$

\subsection{Governing system equations}

The kinetic $\left(E_{k}\right)$ and potential $\left(E_{p}\right)$ energies of the mechanical system read:

$$
\left\{\begin{array}{l}
E_{k}=\frac{1}{2} M\left(\dot{x}_{G}^{2}+\dot{y}_{G}^{2}+\dot{z}_{G}^{2}\right)+\frac{1}{2} I_{y y} \dot{\varphi}^{2}+\frac{1}{2} I_{x x} \dot{\psi}^{2}+\frac{1}{2} m\left(\dot{x}_{m}^{2}+\dot{y}_{m}^{2}+\dot{z}_{m}^{2}\right) \\
E_{p}=g\left(M z_{G}+m z_{m}\right)+\frac{1}{2} K_{y} \psi^{2}+\mathcal{S}(u)
\end{array}\right.
$$




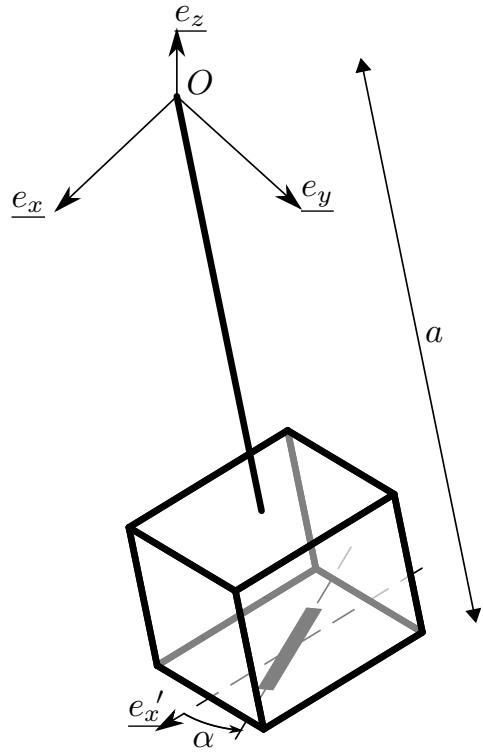

(a) Position and orientation of the absorber on the pendulum.

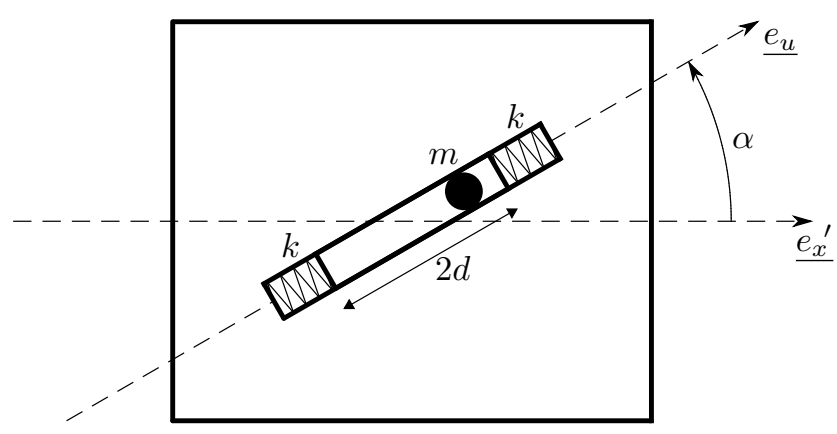

(b) Detailed of the absorber: the mass $m$ moves between two springs with the stiffness $k$ separated by the clearance $2 d$.

Figure 3: Location and representation of the non-smooth absorber.

where $g$ is the local gravitational constant on earth and $\nabla \mathcal{S}(u)=s(u)$, with $\nabla$ stands for the gradient operator. The external and non-conservatives forces applied on the system are:

$$
\begin{aligned}
& F_{\varphi}=-C_{x} \dot{\varphi}+\cos (\beta) f_{0} \sin (\Omega t) \\
& F_{\psi}=-C_{y} \dot{\psi}+\sin (\beta) f_{0} \sin (\Omega t) \\
& F_{u}=-C_{u} \dot{u}
\end{aligned}
$$

The equations of the system are obtained with the Lagrange equations:

$$
\mathbf{M}(X) \ddot{X}+\mathbf{C} \dot{X}+K(\dot{X}, X)+g Z(X)=F
$$

where $X=[\varphi, \psi, u]^{T}$. The expressions of the matrices $\mathbf{M}, \mathbf{C}$ and the vectors $K, Z$ and $F$ are given in the By dividing by the mass of the main system $M$, the equation 11 is expressed with new parameters: $\varepsilon=\frac{m}{M}, j_{x}=\frac{I_{y y}}{M}, j_{y}=\frac{I_{x x}}{M}, c_{x}=\frac{C_{x}}{m}, c_{y}=\frac{C_{y}}{m}, \lambda=\frac{C_{u}}{m}, k_{y}=\frac{K_{y}}{M}$ and $f_{0}=\frac{F_{0}}{M}$. For forthcoming simulations and examples, we use system parameters which are reported in Table 1.

Table 1: List of parameters of the model

\begin{tabular}{ccccccccccc}
\hline$\varepsilon$ & $j_{x}$ & $j_{y}$ & $k_{y}$ & $g$ & $a$ & $L$ & $\lambda$ & $c_{x}$ & $c_{y}$ & $\alpha$ \\
\hline $10^{-4}$ & $20 \mathrm{~m}$ & $20 \mathrm{~m}$ & $0.5 \mathrm{~s}^{-2}$ & $9.81 \mathrm{~m} \mathrm{~s}^{-2}$ & $2 \mathrm{~m}$ & $2 \mathrm{~m}$ & $0.3 \mathrm{~s}^{-1}$ & $5 \mathrm{~s}^{-1}$ & $5 \mathrm{~s}^{-1}$ & $\frac{\pi}{6}$ \\
\hline
\end{tabular}




\section{Treatment of system equations}

Here we assume that the angles $\varphi, \psi$ and the displacement $u$ are small. A rescaling is performed thanks to the parameter $\varepsilon$ :

$$
\begin{aligned}
\varphi & =\varepsilon \tilde{\varphi} \\
\psi & =\varepsilon \tilde{\psi} \\
u & =\varepsilon \tilde{u}
\end{aligned}
$$

We assume also that the amplitude of excitation is small $f_{0}=\varepsilon \tilde{f}_{0}$. This rescaling gives a new definition of the force function:

$$
\tilde{s}(\tilde{u})=\frac{s(u)}{m}= \begin{cases}\varepsilon \tilde{k}(\tilde{u}+\tilde{d}) & \text { if } \tilde{u}<\tilde{d} \\ 0 & \text { if }-\tilde{d} \leq \tilde{u} \leq \tilde{d} \\ \varepsilon \tilde{k}(\tilde{u}-\tilde{d}) & \text { if } \tilde{u}>\tilde{d}\end{cases}
$$

where $\tilde{d}=\frac{d}{\varepsilon}$ and $\tilde{k}=\frac{k}{m}$.

We introduce the complex variables of Manevitch [21] as it follows:

$$
\begin{aligned}
\Phi e^{i \Omega t} & =\dot{\tilde{\varphi}}+i \Omega \tilde{\varphi} \\
\Psi e^{i \Omega t} & =\dot{\tilde{\psi}}+i \Omega \tilde{\psi} \\
U e^{i \Omega t} & =\dot{\tilde{u}}+i \Omega \tilde{u}
\end{aligned}
$$

where $i^{2}=-1$.

A multiple scale method [22] is used to treat system equations. We consider that time $t$ is embedded in several scales linked to each other with the parameter $\varepsilon$ :

$$
\tau_{0}=t, \tau_{1}=\varepsilon^{1} t, \tau_{2}=\varepsilon^{2} t, \ldots
$$

Fast time scale is represented by $\tau_{0}$ whereas slow time scales corresponds to $\tau_{1}, \tau_{2}, \ldots$ The different time scales redefine the derivation operator:

$$
\frac{\mathrm{d}}{\mathrm{d} t}=\frac{\partial}{\partial \tau_{0}}+\varepsilon \frac{\partial}{\partial \tau_{1}}+\varepsilon^{2} \frac{\partial}{\partial \tau_{2}}+\ldots
$$

A Galerkin method is used to keep only the first harmonics. This is carried out for an arbitrary function $h\left(\tau_{0}, \tau_{1}, \tau_{2}, \ldots\right)$ of the system via:

$$
H=\frac{\Omega}{2 \pi} \int_{0}^{\frac{2 \pi}{\Omega}} h\left(\tau_{0}, \tau_{1}, \tau_{2}, \ldots\right) e^{-i \Omega \tau_{0}} \mathrm{~d} \tau_{0}
$$

For evaluating the integral of Eq. 17, we assume that $\Phi, \Psi$ and $U$ are independent of time $\tau_{0}$. This assumption will be verified during the multiple scale analysis of the system or we will search for an asymptotic state as $\tau_{0}$ approaches infinity.

In the sections 3.1 and 3.2 , we will consider system equations at different order of $\varepsilon$ corresponding to different scales of time of system responses. 


\subsection{System behavior at fast time scale: $\varepsilon^{0}$ order of system equations.}

Eq. 11 at $\varepsilon^{0}$ order yield to:

$$
\left\{\begin{array}{l}
\frac{\partial \Phi}{\partial \tau_{0}}+i \frac{\Omega^{2}-\omega_{x}^{2}}{2 \Omega} \Phi=0 \\
\frac{\partial \Psi}{\partial \tau_{0}}+i \frac{\Omega^{2}-\omega_{y}^{2}}{2 \Omega} \Psi=0 \\
a \cos (\alpha) \frac{\partial \Phi}{\partial \tau_{0}}+a \sin (\alpha) \frac{\partial \Psi}{\partial \tau_{0}}+\frac{\partial U}{\partial \tau_{0}}+i \cos (\alpha) \frac{a \Omega^{2}-g}{2 \Omega} \Phi \\
\quad+i \sin (\alpha) \frac{a \Omega^{2}-g}{2 \Omega} \Psi+\frac{i \Omega+\lambda}{2} U+R(U)=0
\end{array}\right.
$$

where $\omega_{x}$ and $\omega_{y}$ are the natural frequencies of the pendulum in both directions:

$$
\begin{aligned}
\omega_{x}^{2} & =\frac{L g}{j_{x}+L^{2}} \\
\omega_{y}^{2} & =\frac{L g+k_{y}}{j_{y}+L^{2}}
\end{aligned}
$$

The expression of the function $R(U)$ calculated with the Eq. 17 applied to the non-smooth function $\tilde{s}$ reads [10]:

$$
\begin{aligned}
R(U) & =\frac{\Omega}{2 \pi} \int_{0}^{\frac{2 \pi}{\Omega}} \tilde{s}\left(\frac{U e^{i \omega \tau_{0}}-U^{*} e^{-i \omega \tau_{0}}}{2 i \Omega}\right) e^{-i \Omega \tau_{0}} \mathrm{~d} \tau_{0} \\
& =-\frac{i \tilde{k} U}{2 \Omega} S(|U|)
\end{aligned}
$$

with

$$
S(|U|)= \begin{cases}0 & \text { if }|U|<D \\ \frac{1}{\pi}\left(2 \arccos \left(\frac{D}{|U|}\right)-\frac{2 D \sqrt{|U|^{2}-D^{2}}}{|U|^{2}}\right) & \text { if }|U| \geq D\end{cases}
$$

where $D=\omega \tilde{d}$.

\subsubsection{Description of the slow invariant manifold of the system}

Both natural frequencies of the main system are assumed to be very close to each other:

$$
\omega_{x}=\omega_{y}+\eta \varepsilon
$$

Let us consider a special case where the frequency of the excitation $\Omega$ is close to both natural frequencies of the main system:

$$
\begin{aligned}
& \Omega=\omega_{x}+\sigma_{x} \varepsilon \\
& \Omega=\omega_{y}+\sigma_{y} \varepsilon
\end{aligned}
$$

Considering the Eqs. 26 and 27 in the Eqs. 18 and 19, one obtains:

$$
\left\{\begin{array}{l}
\frac{\partial \Phi}{\partial \tau_{0}}=0 \\
\frac{\partial \Psi}{\partial \tau_{0}}=0
\end{array}\right.
$$


We conclude, $\Phi$ and $\Psi$ are constant in fast time scale. In this case, the Eq. 20 becomes:

$$
\frac{\partial U}{\partial \tau_{0}}+i \cos (\alpha) \frac{a \omega^{2}-g}{2 \omega} \Phi+i \sin (\alpha) \frac{a \omega^{2}-g}{2 \omega} \Psi+\frac{i \omega+\lambda}{2} U-\frac{i \tilde{k} U}{2 \omega} S(|U|)=0
$$

In the Eq. 29 and in the rest of the article, considering the Eq. 25, the frequency $\omega$ can be equal to $\omega_{x}$ or $\omega_{y}$. Let us seek for an asymptotic state when $\tau_{0}$ approaches infinity. In other words, we are interested in tracing system behavior at its fixed points $\left(\frac{\partial U}{\partial \tau_{0}}=0\right)$. Rewriting the complex variables in polar form as $\Phi=N_{\varphi} e^{i \delta_{\varphi}}, \Psi=N_{\psi} e^{i \delta_{\psi}}$ and $U=N_{u} e^{i \delta_{u}}$ the Eq. 29 yield to the equation of the slow invariant manifold (SIM):

$$
\mathcal{H}=\cos ^{2}(\alpha) N_{\varphi}^{2}+\sin ^{2}(\alpha) N_{\psi}^{2}+2 N_{\varphi} N_{\psi} \sin (\alpha) \cos (\alpha) \cos (\delta)-P^{2}\left(N_{u}\right)=0
$$

where

$$
P^{2}\left(N_{u}\right)=\frac{\left(\tilde{k} S\left(N_{u}\right)-\omega^{2}\right)^{2}+\lambda^{2} \omega^{2}}{\left(a \omega^{2}-g\right)^{2}} N_{u}^{2} \quad \text { and } \quad \delta=\delta_{\varphi}-\delta_{\psi}
$$

The SIM depends on the phase $\delta$. Fig. 4 shows the representation of the SIM for several values of $\delta$ in the space of amplitudes $\left(N_{\varphi}, N_{\psi}, N_{u}\right)$.

\subsubsection{Stability of the SIM}

To determine the stability zones of the SIM, we perturb system variables. In the Eq. 29, we introduce a small perturbation of $U$ as:

$$
U \rightarrow U+\Delta U, \Delta U \ll U
$$

Depending on the value of $N_{u}$, two cases occur:

- Case $N_{u} \leq D$

The linearization of Eqs. 29 considering Eq. 32 leads to:

$$
\left[\begin{array}{c}
\frac{\partial(\Delta U)}{\partial \tau_{0}} \\
\frac{\partial\left(\Delta U^{*}\right)}{\partial \tau_{0}}
\end{array}\right]=\left[\begin{array}{cc}
-\frac{\lambda}{2}-i \frac{\omega}{2} & 0 \\
0 & -\frac{\lambda}{2}+i \frac{\omega}{2}
\end{array}\right]\left[\begin{array}{c}
\Delta U \\
\Delta U^{*}
\end{array}\right]
$$

The real part of both eigenvalues is negative so the system is stable.

- Case $N_{u}>D$

The linearization of Eqs. 29 considering Eq. 32 leads to:

$$
\left[\begin{array}{c}
\frac{\partial(\Delta U)}{\partial \tau_{0}} \\
\frac{\partial\left(\Delta U^{*}\right)}{\partial \tau_{0}}
\end{array}\right]=\underbrace{\left[\begin{array}{cc}
D_{1} & D_{2} \\
D_{2}^{*} & D_{1}^{*}
\end{array}\right]}_{\mathbf{D}}\left[\begin{array}{c}
\Delta U \\
\Delta U^{*}
\end{array}\right]
$$

with

$$
D_{1}=-\frac{\lambda}{2}-\frac{i \omega}{2}+i \frac{\tilde{k} \arccos \left(\frac{D}{N_{u}}\right)}{\pi \omega} \quad \text { and } \quad D_{2}=i \frac{D \tilde{k} \sqrt{N_{u}^{2}-D^{2}}}{\omega \pi U^{* 2}}
$$

and the symbol ${ }^{*}$ represents the complex conjugate of the concerned function. The characteristic polynomial of the matrix $\mathbf{D}$ reads:

$$
P_{\text {car }}(X)=X^{2}-\left(D_{1}+D_{1}^{*}\right) X+\left|D_{1}\right|^{2}-\left|D_{2}\right|^{2}
$$




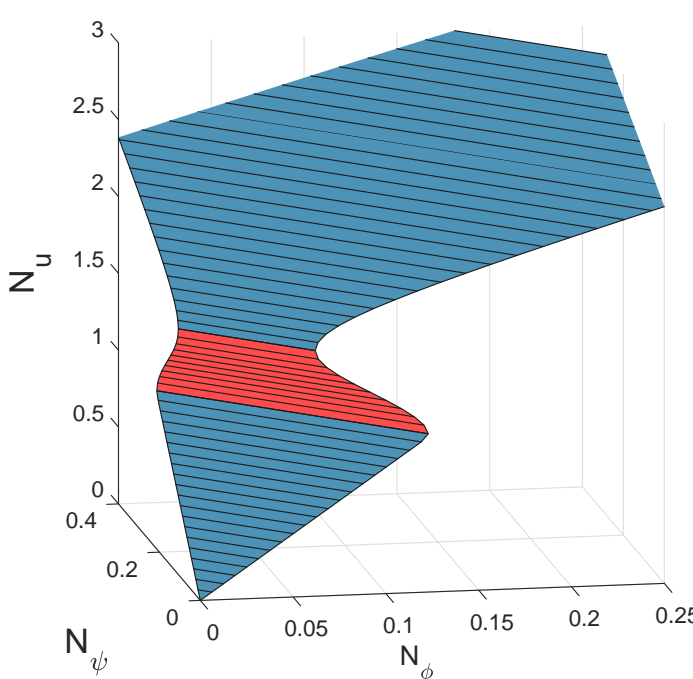

(a) $\delta=0$

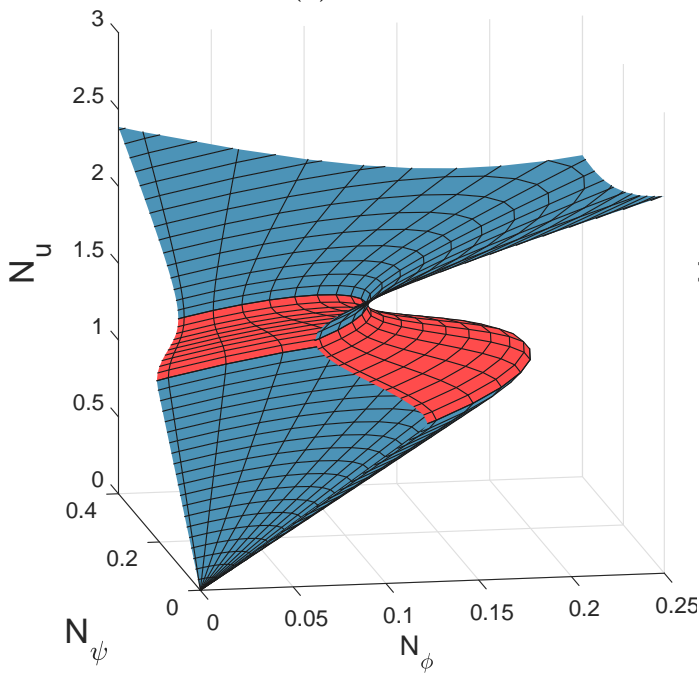

(c) $\delta=0.8 \pi$

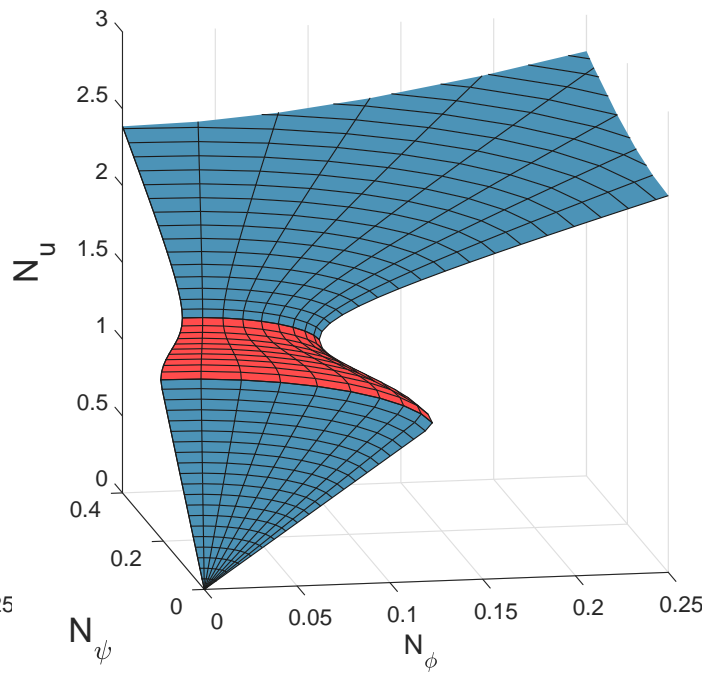

(b) $\delta=\frac{\pi}{2}$

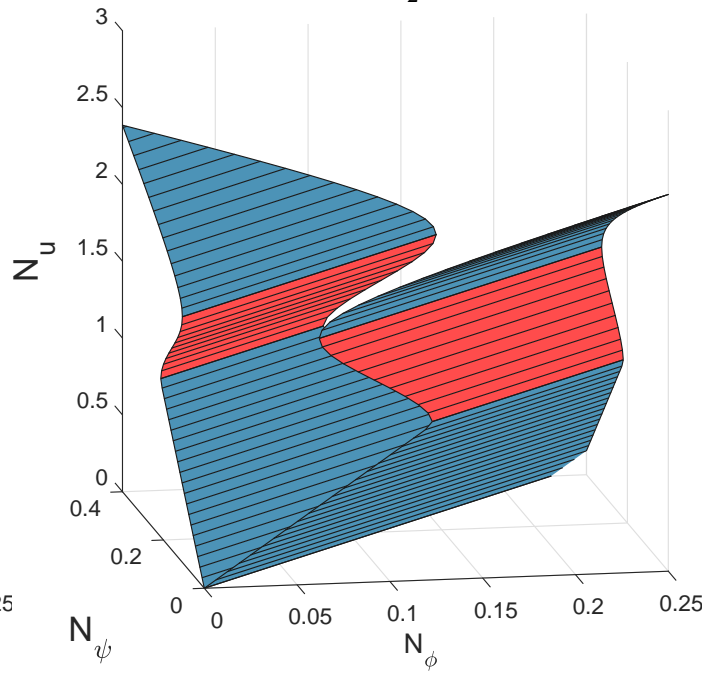

(d) $\delta=\pi$

Figure 4: The SIM of the system for several values of $\delta$ in the amplitude space $\left(N_{\varphi}, N_{\psi}, N_{u}\right)$. The zones in blue are stable whereas the zones in red are unstable.

The sum $S_{\mathbf{D}}$ and the product $P_{\mathbf{D}}$ of the eigenvalues of the matrix $\mathbf{D}$ read:

$$
\begin{aligned}
& S_{\mathbf{D}}=D_{1}+D_{1}^{*}=-\lambda<0 \\
& P_{\mathbf{D}}=\left|D_{1}\right|^{2}-\left|D_{2}\right|^{2}
\end{aligned}
$$

We can deduct that the SIM is unstable if $\left|D_{1}\right|^{2}-\left|D_{2}\right|^{2}<0$, i.e.:

$$
\frac{c^{2}}{4}+\frac{\omega^{2}}{4}-\frac{k_{l} \arccos \left(\frac{D}{N_{u}}\right)}{\pi}+\frac{k_{l}^{2} \arccos \left(\frac{D}{N_{u}}\right)^{2}}{\omega^{2} \pi^{2}}-\frac{D^{2} k_{l}^{2}}{N_{u}^{2} \omega^{2} \pi^{2}}+\frac{D^{4} k_{l}^{2}}{N_{u}^{4} \omega^{2} \pi^{2}}<0
$$

The equation 39 leads to:

$$
\sin ^{2}(Y)>\left(Y-\frac{\omega^{2} \pi}{\tilde{k}}\right)^{2}+\left(\frac{\pi \lambda \omega}{\tilde{k}}\right)^{2}
$$


with $Y=2 \arccos \left(\frac{D}{N_{u}}\right)$. The unstable zone, represented in red on Fig. 4, does not depend on the amplitudes $N_{\varphi}$ and $N_{\psi}$.

\subsection{System behavior at slow time scale: $\varepsilon^{1}$ order of system equation.}

The first two equations of the system 11 at $\varepsilon^{1}$ order read:

$$
\left\{\begin{array}{c}
L g \frac{\partial \Phi}{\partial \tau_{1}}+\left(\frac{\omega^{2} c_{x}}{2}+i \frac{2 \sigma_{x} L g+a \omega\left(a \omega^{2}-g\right)}{2}\right) \Phi \\
+i \cos (\alpha) \frac{\omega\left(a \omega^{2}-g\right)}{2} U+\frac{i \tilde{f}_{0} \cos (\beta) \omega^{2}}{2}=0 \\
\left(L g+k_{y}\right) \frac{\partial \Psi}{\partial \tau_{1}}+\left(\frac{\omega^{2} c_{y}}{2}+i \frac{2 \sigma_{y} L g+a \omega\left(a \omega^{2}-g\right)}{2}\right) \Psi \\
\quad+i \sin (\alpha) \frac{\omega\left(a \omega^{2}-g\right)}{2} U+\frac{i \tilde{f}_{0} \sin (\beta) \omega^{2}}{2}=0
\end{array}\right.
$$

or in a compact form, Eqs. 41 and 42 can be written as:

$$
\frac{\partial \Phi}{\partial \tau_{1}}=f_{\varphi}(\Phi, \Psi, U) ; \quad \frac{\partial \Psi}{\partial \tau_{1}}=f_{\psi}(\Phi, \Psi, U)
$$

To seek system responses around the SIM, we need to trace equilibrium and singular points. To find the equilibrium points of the system we have to solve the system of equations formed by the Eqs. 41 and 42 and the evolution of the SIM at $\tau_{1}$ time scale [8]:

$$
\left\{\begin{array}{l}
f_{\varphi}(\Phi, \Psi, U)=0 \\
f_{\psi}(\Phi, \Psi, U)=0 \\
\left(\omega^{2}-i \omega \lambda\right) U-i \tilde{k} U S(|U|)+\cos (\alpha)\left(a \omega^{2}-g\right) \Phi+\sin (\alpha)\left(a \omega^{2}-g\right) \Psi=0 \\
\operatorname{det}\left(\nabla_{\left(U, U^{*}\right)} \mathcal{H}\right) \neq 0
\end{array}\right.
$$

where $\nabla_{\left(U, U^{*}\right)} \mathcal{H}$ represents the Jacobian matrix of $\mathcal{H}$ versus variables $U$ and $U^{*}$.

The expression of $\Phi$ and $\Psi$ can be found as a function of $U$ from the Eqs. 44 and 45 :

$$
\left\{\begin{array}{l}
\Phi=-\frac{\cos (\alpha)\left(a \omega^{2}-g\right) U+\omega \tilde{f}_{0} \cos (\beta)}{2 L g \sigma_{x} / \omega+a^{2} \omega^{2}-a g-i c_{x} \omega} \\
\Psi=-\frac{\sin (\alpha)\left(a \omega^{2}-g\right) U+\omega \tilde{f}_{0} \sin (\beta)}{2 L g \sigma_{y} / \omega+a^{2} \omega^{2}-a g-i c_{y} \omega}
\end{array}\right.
$$

We assume a priori that $N_{u} \leq d$, by replacing the Eqs. 48 and 49 in the Eq. 46, one obtains:

$$
U=\frac{C \tilde{f}_{0}}{\left(\omega^{2}-i \omega \lambda+A+i B\right)}
$$

where:

$$
\begin{gathered}
A=\operatorname{Re}\left(-\frac{\cos ^{2}(\alpha)\left(a \omega^{2}-g\right)^{2}}{2 L g \sigma_{x} / \omega+a^{2} \omega^{2}-a g-i c_{x} \omega}-\frac{\sin ^{2}(\alpha)\left(a \omega^{2}-g\right)^{2}}{2 L g \sigma_{y} / \omega+a^{2} \omega^{2}-a g-i c_{y} \omega}\right) \\
B=\operatorname{Im}\left(-\frac{\cos ^{2}(\alpha)\left(a \omega^{2}-g\right)^{2}}{2 L g \sigma_{x} / \omega+a^{2} \omega^{2}-a g-i c_{x} \omega}-\frac{\sin ^{2}(\alpha)\left(a \omega^{2}-g\right)^{2}}{2 L g \sigma_{y} / \omega+a^{2} \omega^{2}-a g-i c_{y} \omega}\right) \\
C=-\frac{\cos (\alpha)\left(a \omega^{2}-g\right) \omega \cos (\beta)}{2 L g \sigma_{x} / \omega+a^{2} \omega^{2}-a g-i c_{x} \omega}-\frac{\sin (\alpha)\left(a \omega^{2}-g\right) \omega \sin (\beta)}{2 L g \sigma_{y} / \omega+a^{2} \omega^{2}-a g-i c_{y} \omega}
\end{gathered}
$$


We can now verify the assumption $N_{u} \leq d$. If it is false, we have to consider that $N_{u}>d$. The amplitude $U$ can be expressed with its modulus and argument as $U=N_{u} e^{i \delta_{u}}$. Eq. 50 reads:

$$
\left[-\tilde{k} N_{u} S\left(N_{u}\right)+\left(\omega^{2}+A+i(B-\omega \lambda)\right) N_{u}\right] e^{i \delta_{u}}=-C \tilde{f}_{0}
$$

Considering the module of both parts of Eq. 54, we obtain:

$$
\left(-\tilde{k} N_{u} S\left(N_{u}\right)+\left(\omega^{2}+A\right) N_{u}\right)^{2}+(B-\omega \lambda)^{2} N_{u}^{2}-|C|^{2} \tilde{f}_{0}^{2}=0
$$

The Eq. 55 is solved numerically.

The role of Eq. 47 is to determine if the solution of the system is an equilibrium point or a singular point. Let us first evaluate $\nabla_{\left(U, U^{*}\right)} \mathcal{H}$. Considering the complex conjugate of the equation of the SIM (see Eq. 30), we will have:

$$
\begin{aligned}
\nabla_{\left(U, U^{*}\right)} \mathcal{H} & =\left[\begin{array}{cc}
\frac{\partial \mathcal{H}}{\partial U} & \frac{\partial \mathcal{H}}{\partial U^{*}} \\
\frac{\partial \mathcal{H}^{*}}{\partial U} & \frac{\partial \mathcal{H}^{*}}{\partial U^{*}}
\end{array}\right] \\
& =\left[\begin{array}{cc}
\frac{\lambda+i \omega}{2}-i \frac{\tilde{k} \arccos \left(\frac{D}{N_{u}}\right)}{\omega \pi} & -i \frac{\tilde{k} D \sqrt{N_{u}^{2}-D^{2}}}{U^{* 2} \omega \pi} \\
i \frac{\tilde{k} D \sqrt{N_{u}^{2}-D^{2}}}{U^{2} \omega \pi} & \frac{\lambda-i \omega}{2}+i \frac{\tilde{k} \arccos \left(\frac{D}{N_{u}}\right.}{\omega \pi}
\end{array}\right]
\end{aligned}
$$

The matrix $\mathbf{D}$ is the same as the matrix of Eq. 34 used to determine stability zones of the SIM. Thus, all the points located on the border of the unstable zone of the SIM are singular. They are solutions of the following equation [23]:

$$
\sin ^{2}(Y)=\left(Y-\frac{\omega^{2} \pi}{\tilde{k}}\right)^{2}+\left(\frac{\pi \lambda \omega}{\tilde{k}}\right)^{2}
$$

Fig. 5 shows the values of $N_{\varphi}, N_{\psi}$ and $N_{u}$ of the equilibrium points of the system as a function of $\omega$ for $\tilde{f}_{0}=20$. The oscillation amplitudes around $\underline{e_{y}}$ (angle $\varphi$ ) are more reduced than the ones around $\underline{e_{y}}(\psi)$. This can be explained by the direction of the absorber $\alpha=\frac{\pi}{6}$. Its efficiency is more important on the $\underline{e}_{x}$ direction. An isolated branch exists with high levels of $N_{\varphi}$. This isolated branch is dangerous because it can attract the system to a state with significant level of $\varphi$.

\section{Numerical study}

Here, the asymptotic results are confronted with numerical ones obtained by direct numerical integration of the Eq. 11. The aim is to validate the assumptions made in the analytic study and to determine the level of efficiency of the absorber in different configurations. Three time integrations of the system are made with different frequency of excitation $\Omega$ to compare with the analytic results. The amplitude of the force is the same as the one of the Fig. 5 i.e. $\tilde{f}_{0}=20$.

As the first example we consider the system with $\Omega=0.903 \mathrm{rads}^{-1}$ corresponding to the frequency which can bring the system to possess an isolated branch (see Fig 5). At this frequency, three stable equilibrium points exist. The results depicted in Fig. 6 show that the system is going to the equilibrium point with the highest amplitude of $N_{\varphi}$. The isolated 


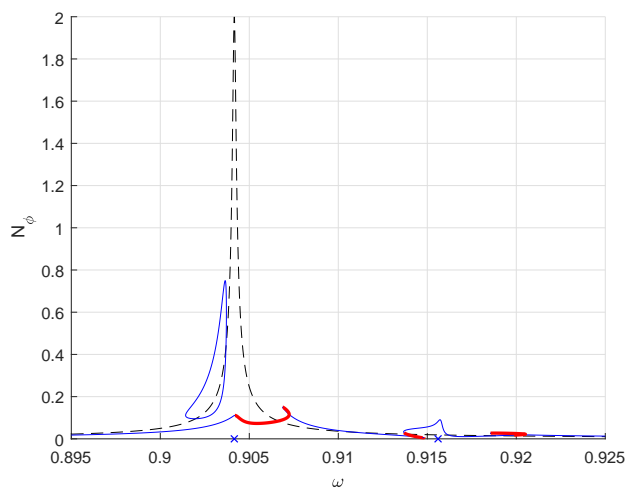

(a) $N_{\varphi}$

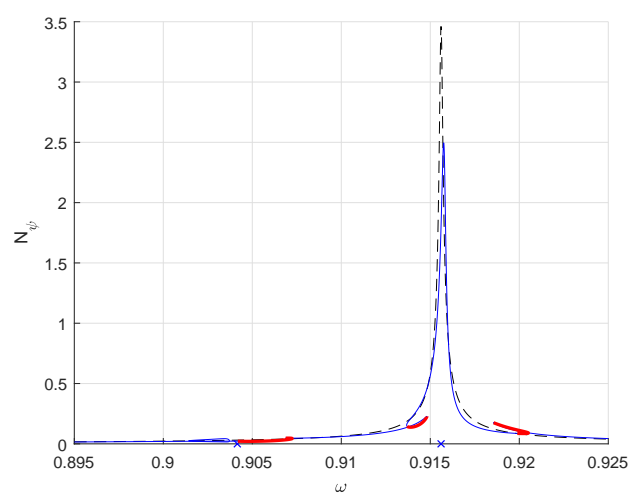

(b) $N_{\psi}$

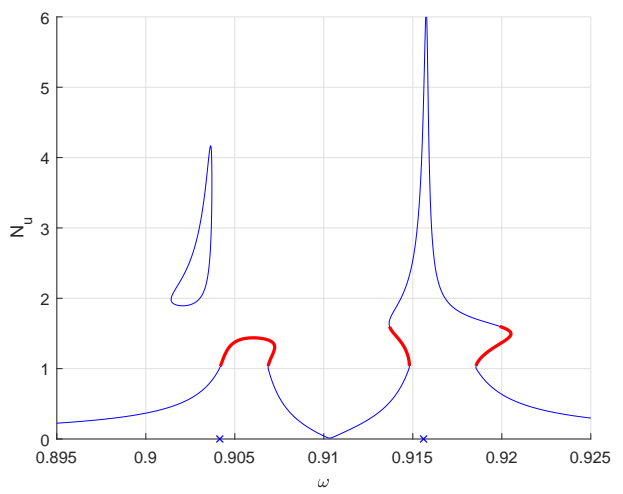

(c) $N_{u}$

Figure 5: Amplitude of the equilibrium points as a function of $\omega$ for $\tilde{f}_{0}=20$. Blue line $(-)$ is stable whereas red line $(-)$ is unstable. Crosses $(x)$ represent the natural frequencies of the main system i.e. $\omega_{x}$ and $\omega_{y}$. The dashed lines (- - ) correspond to the response of the system without NES.

branches with high energy level can be problematic as they can bring the main system to oscillate with high amplitude.

Let us consider the system with $\Omega=0.905 \mathrm{rad} \mathrm{s}^{-1}$ : the only equilibrium point is unstable because it is located in the unstable zone of the SIM. As shown on Fig 7, the system is oscillating between the stables zones due to the singular points. When the system reaches a singular point, the amplitude of the displacement of the absorber $N_{u}$ jump to another point of the SIM which can be relatively far.

These oscillations are called SMR [7]. They are used for keeping the amplitude of the oscillations of the main system, here $N_{\varphi}$ and $N_{\psi}$, below a threshold. The value of this threshold is given by the coordinates of the first singular point that is a solution of the equation 57. For $N_{\varphi}$, it is equal to 0.125 [24]

As the third example, let us set $\Omega=0.917 \mathrm{rad} \mathrm{s}^{-1}$ which is close to the natural frequency $\omega_{y}$. Figure 8 shows that the amplitude $N_{\psi}$ reaches high values. This is coherent with the analytic calculation of equilibrium points represented on Fig. 5. The only equilibrium point is in the second stable zone of the SIM because the value of $\tilde{f}_{0}$ is too high. The highest value of $\tilde{f}_{0}$ to keep the amplitude $N_{\psi}$ below the threshold of 0.216 can be determined numerically with the equation 55 . It is equal to 10.58 . 


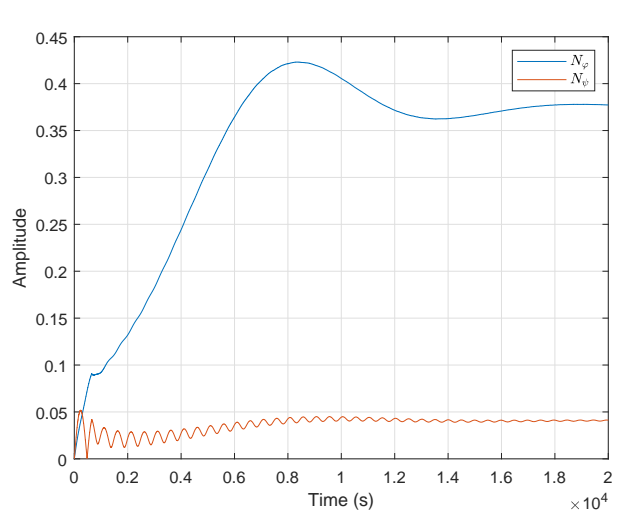

(a) $N_{\varphi}$ and $N_{\psi}$

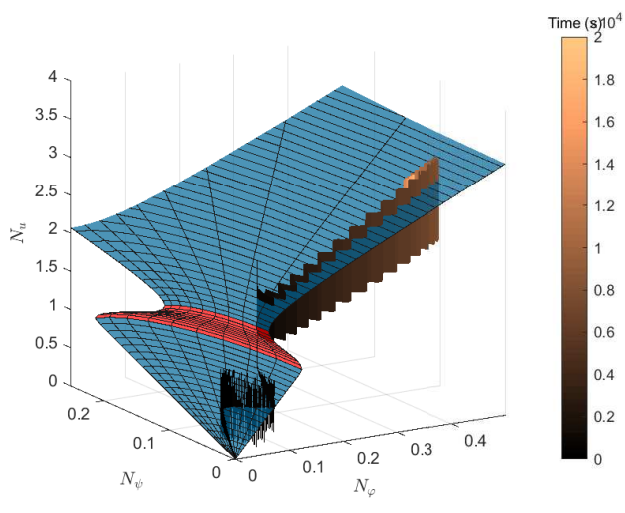

(b) SIM versus numerical results

Figure 6: (a) Time histories of the system amplitudes, i.e., $N_{\varphi}$ and $N_{\psi}$. (b) The SIM versus numerically obtained amplitudes. Numerical results are obtained via direct integration of Eq. 11 for the system with $\tilde{f}_{0}=20$ and $\Omega=0.903 \mathrm{rad} \mathrm{s}^{-1}$.

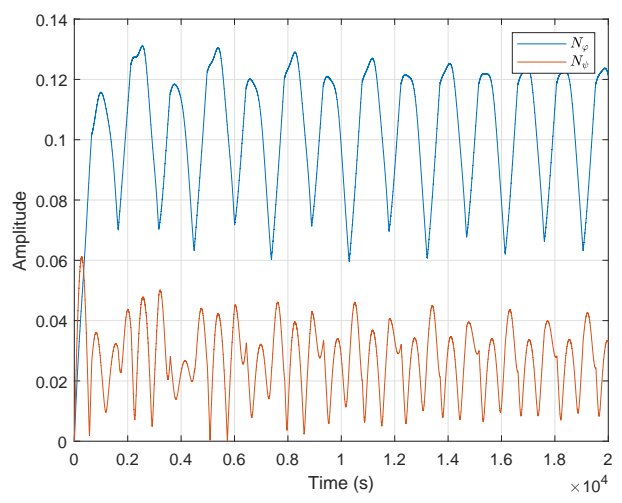

(a) $N_{\varphi}$ and $N_{\psi}$

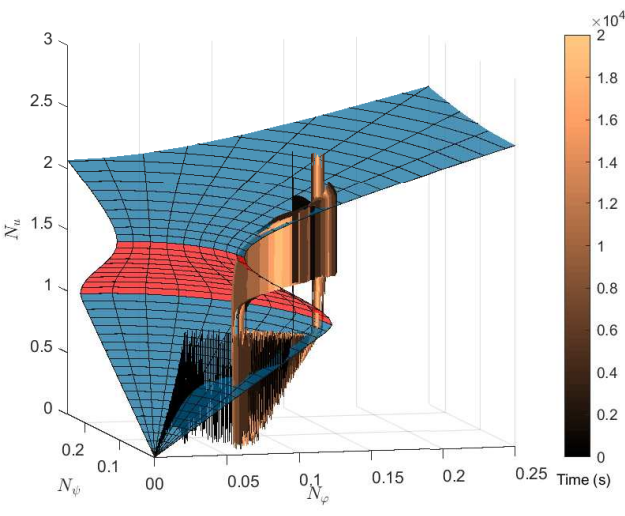

(b) SIM versus numerical results

Figure 7: (a) Time histories of the system amplitudes, i.e., $N_{\varphi}$ and $N_{\psi}$. (b) The SIM versus numerically obtained amplitudes. Numerical results are obtained via direct integration of Eq. 11 for the system with $\tilde{f}_{0}=20$ and $\Omega=0.905 \mathrm{rad} \mathrm{s}^{-1}$.

\section{Conclusion}

A nonlinear non-smooth absorber is coupled to a two degree-of-freedom pendulum at an arbitrary location and orientation. The non-smooth absorber possesses a piece-wise linear restoring forcing function, representing two elastic barriers separated with the given clearance. A generalized force is applied to the system in a certain direction. The multiple scales method allows the system to be analyzed at different orders of time. At fast time scale, the behavior of the system is described by a slow invariant manifold in the space of amplitude of the three degrees of freedom. The slow invariant manifold depends on the phase between both angles of the main system. The stable and unstable zones are also highlighted. At slow time scale, the equilibrium points of the system are calculated by solving numerically an analytic equation.

The numerical study bring out the important parameters of the conception of the absorber to be efficient to reduce vibration of the main system. 


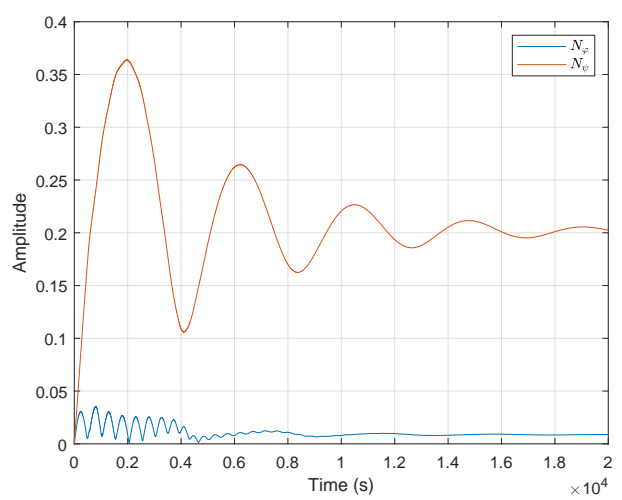

(a) $N_{\varphi}$ and $N_{\psi}$

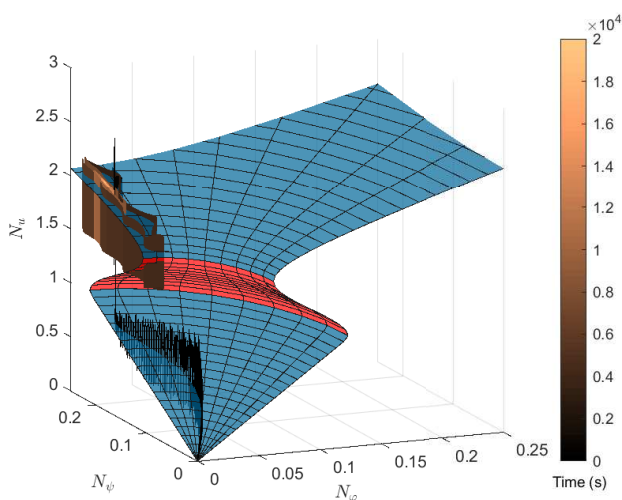

(b) SIM versus numerical results

Figure 8: (a) Time histories of the system amplitudes, i.e., $N_{\varphi}$ and $N_{\psi}$. (b) The SIM versus numerically obtained amplitudes. Numerical results are obtained via direct integration of Eq. 11 for the system with $\tilde{f}_{0}=20$ and $\Omega=0.917 \mathrm{rad} \mathrm{s}^{-1}$.

Acknowledgments The authors would like to thank "La Region Auvergne-Rhone-Alpes" for supporting this work in the frame of the CALIPSO project.

\section{Appendix}

The expression of the matrices and vectors of the dynamic equation 11 read:

$$
\mathbf{M}=\left[\begin{array}{lll}
m_{11} & m_{12} & m_{13} \\
m_{21} & m_{22} & m_{23} \\
m_{31} & m_{32} & m_{33}
\end{array}\right]
$$

with

$$
\begin{aligned}
& m_{11}=L^{2}+j_{x}+\varepsilon\left(a^{2}+u^{2} \cos ^{2}(\alpha)\right) \\
& m_{12}=m_{21}=\varepsilon\left[\sin (\varphi) a u+\cos (\alpha) \cos (\varphi) u^{2}\right] \sin (\alpha) \\
& m_{13}=m_{31}=a \varepsilon \cos (\alpha) \\
& m_{22}=j_{y}+L^{2} \cos ^{2}(\varphi)+\varepsilon\left[u^{2}-u^{2} \cos ^{2}(\alpha) \cos ^{2}(\varphi)\right. \\
& \left.\quad-2 a u \cos (\alpha) \cos (\varphi) \sin (\varphi)+a^{2} \cos ^{2}(\varphi)\right] \\
& m_{23}=m_{32}=\varepsilon a \sin (\alpha) \cos (\varphi) \\
& m_{33}=\varepsilon
\end{aligned}
$$


with

$$
\begin{aligned}
k_{1} & =\left[\sin (\varphi) L^{2} \cos (\varphi)+\varepsilon \sin (\varphi) a^{2} \cos (\varphi)-\varepsilon a u \cos (\alpha)\right. \\
& \left.+2 \varepsilon a \cos (\alpha) \cos ^{2}(\varphi) u-\varepsilon \sin (\varphi) u^{2} \cos ^{2}(\alpha) \cos (\varphi)\right] \dot{\psi}^{2} \\
& +2 \varepsilon[a \sin (\alpha) \sin (\varphi)+\sin (\alpha) \cos (\alpha) \cos (\varphi) u] \dot{\psi} \dot{u}+2 \varepsilon \cos ^{2}(\alpha) u \dot{\varphi} \dot{u} \\
k_{2} & =\left[a \varepsilon u \cos (\varphi) \sin (\alpha)-\varepsilon u^{2} \cos (\alpha) \sin (\alpha) \sin (\varphi)\right] \dot{\varphi}^{2} \\
& +\left[-2 L^{2} \cos (\varphi) \sin (\varphi)-2 \varepsilon\left(a^{2} \cos (\varphi) \sin (\varphi)+a u \cos (\alpha)\right.\right. \\
& \left.+u^{2} \cos ^{2}(\alpha) \cos (\varphi) \sin (\varphi)-2 a u \cos (\alpha) \cos ^{2}(\varphi)\right) \dot{\varphi} \dot{\psi} \\
& +2 \varepsilon u \cos (\alpha) \cos (\varphi) \sin (\alpha) \dot{\varphi} \dot{u} \\
& +2 \varepsilon\left[u-a \cos (\alpha) \cos (\varphi) \sin (\varphi)-u \cos ^{2}(\alpha) \cos ^{2}(\varphi)\right] \dot{\psi} \dot{u}+k_{y} \psi \\
k_{3}= & \varepsilon\left[\left(a \cos (\alpha) \cos (\varphi) \sin (\varphi)-u+u \cos ^{2}(\alpha) \cos ^{2}(\varphi)\right) \dot{\psi}^{2}\right. \\
& -2(a \sin (\alpha) \sin (\varphi)+u \cos (\alpha) \cos (\varphi) \sin (\alpha)) \dot{\varphi} \dot{\psi} \\
& \left.-\cos ^{2}(\alpha) \dot{\varphi}^{2} u+\frac{s(u)}{m}\right] \quad Z=\left[\begin{array}{c}
z_{1} \\
z_{2} \\
z_{3}
\end{array}\right]
\end{aligned}
$$

with

$$
\begin{aligned}
& z_{1}=L \sin (\varphi) \cos (\psi)+\varepsilon[a \sin (\varphi) \cos (\psi)+\cos (\alpha) \cos (\varphi) \cos (\psi) u] \\
& z_{2}=L \cos (\varphi) \sin (\psi) \\
& +\varepsilon[a \cos (\varphi) \sin (\psi)+u \cos (\psi) \sin (\alpha)-u \cos (\alpha) \sin (\varphi) \sin (\psi)] \\
& z_{3}=\varepsilon[\sin (\alpha) \sin (\psi)+\cos (\alpha) \cos (\psi) \sin (\varphi)] \\
& F=\varepsilon f_{0} \sin (\Omega t)\left[\begin{array}{c}
\cos (\beta) \\
\sin (\beta) \\
0
\end{array}\right]
\end{aligned}
$$

\section{References}

[1] R.A. Ibrahim. "Recent advances in nonlinear passive vibration isolators". Journal of Sound and Vibration 314.3-5 (2008), pp. 371-452. DOI: 10.1016/j.jsv.2008.01.014 (cit. on p. 1).

[2] Hermann Frahm. "Device for damping vibrations of bodies." Pat. US Patent 989,958. 1911 (cit. on p. 1).

[3] J. P. Den Hartog. Mechanical vibrations. New York, 1956 (cit. on p. 1).

[4] Robert E. Roberson. "Synthesis of a nonlinear dynamic vibration absorber". Journal of the Franklin Institute 254.3 (1952), pp. 205-220. DOI: 10.1016/0016-0032(52)90457-2 (cit. on p. 1).

[5] O. Gendelman, L. I. Manevitch, A. F. Vakakis, and R. M'Closkey. "Energy Pumping in Nonlinear Mechanical Oscillators: Part I-Dynamics of the Underlying Hamiltonian Systems". Journal of Applied Mechanics 68.1 (2001), p. 34. DOI: 10.1115/1.1345524 (cit. on p. 1).

[6] A. F. Vakakis and O. Gendelman. "Energy Pumping in Nonlinear Mechanical Oscillators: Part II. Resonance Capture". Journal of Applied Mechanics 68.1 (2001), p. 42. DOI: $10.1115 / 1.1345525$ (cit. on p. 1). 
[7] Y. Starosvetsky and O.V. Gendelman. "Strongly modulated response in forced 2DOF oscillatory system with essential mass and potential asymmetry". Physica D: Nonlinear Phenomena 237.13 (2008), pp. 1719-1733. DOI: 10.1016/j.physd.2008.01.019 (cit. on pp. 1, 11).

[8] A. Ture Savadkoohi, C.-H. Lamarque, M. Weiss, B. Vaurigaud, and S. Charlemagne. "Analysis of the 1:1 resonant energy exchanges between coupled oscillators with rheologies". Nonlinear Dynamics 86.4 (2016), pp. 2145-2159. DOI: 10.1007/s11071-0162792-3 (cit. on pp. 1, 9).

[9] F. Nucera, A. F. Vakakis, D. M. McFarland, L. A. Bergman, and G. Kerschen. "Targeted energy transfers in vibro-impact oscillators for seismic mitigation". Nonlinear Dynamics 50.3 (2007), pp. 651-677. DOI: 10.1007/s11071-006-9189-7 (cit. on p. 1).

[10] Oleg V. Gendelman. "Targeted energy transfer in systems with non-polynomial nonlinearity". Journal of Sound and Vibration 315.3 (2008), pp. 732-745. DOI: 10.1016/j. jsv.2007.12.024 (cit. on pp. 1, 6).

[11] I Karayannis, A F Vakakis, and F Georgiades. "Vibro-impact attachments as shock absorbers". Proceedings of the Institution of Mechanical Engineers, Part C: Journal of Mechanical Engineering Science 222.10 (2008), pp. 1899-1908. DOI: 10.1243/ $09544062 J M E S 864$ (cit. on p. 1).

[12] C.-H. Lamarque, O. V. Gendelman, A. Ture Savadkoohi, and E. Etcheverria. "Targeted energy transfer in mechanical systems by means of non-smooth nonlinear energy sink". Acta Mechanica 221.1-2 (2011), pp. 175-200. DOI: 10.1007/s00707-011-0492-0 (cit. on p. 1).

[13] Mohammad A. AL-Shudeifat, Nicholas Wierschem, D. Dane Quinn, Alexander F. Vakakis, Lawrence A. Bergman, and Billie F. Spencer. "Numerical and experimental investigation of a highly effective single-sided vibro-impact non-linear energy sink for shock mitigation". International Journal of Non-Linear Mechanics 52 (2013), pp. 96109. DOI: 10.1016/j.ijnonlinmec.2013.02.004 (cit. on p. 1).

[14] O.V. Gendelman and A. Alloni. "Dynamics of forced system with vibro-impact energy sink". Journal of Sound and Vibration 358 (2015), pp. 301-314. DOI: 10.1016/j.jsv. 2015.08.020 (cit. on p. 1).

[15] Alireza Ture Savadkoohi, Claude-Henri Lamarque, and Zoran Dimitrijevic. "Vibratory energy exchange between a linear and a nonsmooth system in the presence of the gravity". Nonlinear Dynamics 70.2 (2012), pp. 1473-1483. DOI: 10.1007/s11071-0120548-2 (cit. on p. 1).

[16] Hiroshi Matsuhisa and Masashi Yasuda. "Dynamic vibration absorber for pendulum type structure". Pat. US Patent 5,460,099. 1995 (cit. on p. 1).

[17] Hiroshi Matsuhisa, Rongrong Gu, Yongjing Wang, Osamu Nishihara, and Susumu Sato. "Vibration Control of a Ropeway Carrier by Passive Dynamic Vibration Absorbers". JSME international journal. Ser. C, Dynamics, control, robotics, design and manufacturing 38.4 (1995), pp. 657-662. DOI: 10.1299/jsmec1993.38.657 (cit. on p. 1).

[18] Y. Starosvetsky and O. V. Gendelman. "Dynamics of a strongly nonlinear vibration absorber coupled to a harmonically excited two-degree-of-freedom system". Journal of Sound and Vibration 312.1 (2008), pp. 234 -256. DOI: 10.1016/j.jsv.2007.10.035 (cit. on p. 1).

[19] Thanh Tung Pham, Claude-Henri Lamarque, and Alireza Ture Savadkoohi. "Multiresonance capturing in a two-degree-of-freedom system under two different harmonic excitations". Journal of Vibration and Control 18.3 (2012), pp. 451-466. DOI: 10.1177/ 1077546311404268 (cit. on p. 1). 
[20] Gabriel Hurel, Alireza Ture Savadkoohi, and Claude-Henri Lamarque. "Nonlinear vibratory energy exchanges between a two degrees-of-freedom pendulum and a nonlinear absorber." Journal of Engineering Mechanics (2019). DOI: 10.1061/(ASCE)EM.19437889.0001620 (cit. on p. 1).

[21] L. I. Manevitch. "The Description of Localized Normal Modes in a Chain of Nonlinear Coupled Oscillators Using Complex Variables". Nonlinear Dynamics 25.1 (2001), pp. 95-109. DOI: 10.1023/A:1012994430793 (cit. on p. 5).

[22] Ali Hasan Nayfeh and Dean T. Mook. Nonlinear oscillations. Wiley classics library ed. Wiley classics library. New York, 1995 (cit. on p. 5).

[23] C.-H. Lamarque, A. Ture Savadkoohi, S. Charlemagne, and P. Abdoulhadi. "Nonlinear vibratory interactions between a linear and a non-smooth forced oscillator in the gravitational field". Mechanical Systems and Signal Processing 89 (2017), pp. 131-148. DOI: 10.1016/j.ymssp.2016.09.043 (cit. on p. 10).

[24] Mathieu Weiss, Bastien Vaurigaud, Alireza Ture Savadkoohi, and Claude-Henri Lamarque. "Control of vertical oscillations of a cable by a piecewise linear absorber". Journal of Sound and Vibration 435 (2018), pp. 281-300. DOI: 10.1016/j.jsv.2018.07. 033 (cit. on p. 11). 